\title{
La vulneración de los derechos patrimoniales de los integrantes de las uniones de hecho por falta de un registro municipal de uniones de hecho en la Provincia de Tacna, 2011
}

\author{
Infringement of proprietary rights of members of unions in fact for lack of a municipal union's \\ record made in the Province of Tacna, 2011
}

\section{Sandra Miranda Pérez ${ }^{1}$}

\section{RESUMEN}

Objetivo: Determinar la forma en que se afectan los derechos patrimoniales y seguridad económica de los concubinos y sus familias al no estar incorporados en un registro municipal de uniones de hecho. Así como, demostrar la necesidad de regular a través de medios alternativos para que se pueda mejorar la forma de probar la relación convivencial, un medio idóneo que pudiera ser el registro municipal.

Método: Para investigar los casos se ha empleado la técnica de la encuesta. Y el instrumento para obtener los datos requeridos para llevar a cabo el desarrollo de la investigación fue obtenido a través de cuestionarios. La muestra fue tomada durante el mes de febrero del 2013 en la ciudad de Tacna, desde el 02 de febrero hasta el 26 de febrero. La investigación corresponde al tipo de investigación aplicada, debido a que tiene como propósito modificar parte del ordenamiento jurídico, por tanto, la finalidad de la investigación es tratar de proporcionar elementos para modificar la realidad.

Resultado: Los resultados confirman que existe una relación significativa entre la falta de un registro de las parejas que mantienen una unión de hecho y la vulneración de los derechos fundamentales patrimoniales de los concubinos; por cuanto, como se verifica la falta de precisión, de integralidad, de precisión de límites en la determinación del inicio del tiempo para el reconocimiento de la relación de unión de hecho, conspira contra los derechos patrimoniales de los concubinos. Por lo tanto, la falta de un registro de las parejas que mantienen una unión de hecho vulnera los derechos fundamentales patrimoniales de los concubinos, por lo que la propuesta viable de un Registro Municipal de Uniones de hecho resultaría un instrumento eficaz para cautelar los derechos patrimoniales de los concubinos.

Conclusiones: Los resultados han permitido constatar que existe una relación significativa entre la falta de un registro de las parejas que mantienen una unión de hecho y la vulneración de los derechos fundamentales patrimoniales de los concubinos.

\section{PALABRAS CLAVE}

Unión de hecho, derechos patrimoniales, sociedad de gananciales, registro civil, registros públicos.

\begin{abstract}
Objective: Determine how they affect the economic rights and economic security of the cohabitants and their families not being incorporated into a municipal register of unions. And demonstrate the need for regulation through alternative means that can improve the way you test the convivial relationship, could be an ideal means the municipal register. Methods: To investigate the cases we have used the survey technique. And the instrument to obtain the data required to carry out the development of the research was obtained through questionnaires. The sample was taken during the month of February 2013 in the city of Tacna, from February 02 to February 26. The research is the type of applied research, because change aims of the legal system, so the aim of the research is to try to provide items to alter reality. The research design to be measured by its location in time is a cross-sectional research.

Results: The results presented confirm that there is a significant relationship between the lack of a record of couples who maintain a common-law and the violation of fundamental property rights of cohabitants, since the lack of accuracy, comprehensiveness of verified limits accuracy in determining the start time for the recognition of common-law relationship conspires against the property rights of cohabitants. Therefore, the lack of a record of couples who maintain a de facto union violates his fundamental property rights of cohabitants, so that a viable proposal Municipal Unions Registration indeed prove an effective instrument to safeguard the property rights of cohabitants.

Conclusions: The results allowed to confirm that there is a significant relationship between the lack of a record of couples who maintain a common-law and the violation of fundamental property rights of cohabitants.
\end{abstract}

\section{KEYWORDS}

Union made, economic rights, community property, civil registration, public registration.

1. Magister en Derecho con mención en Derecho Civil y Comercial, Abogado. Actualmente labora en la Corte Superior de Justicia de Tacna, como Secretaria de la Sala y Administradora del Módulo Laboral de Tacna. Tacna -Perú 


\section{Introducción}

Las uniones de hecho son un fenómeno cada vez más recurrente en la sociedad, no solo en nuestro país sino en otros países. Lo que ayer fue una excepción, hoy es la generalidad. Las uniones de hecho, según Cornejo, M. T. (2000), ya no constituyen un estigma como lo fue en el pasado aunque todavía existen sectores de la sociedad que miran este tipo de familia con prejuicio. Fue el Tribunal Constitucional el primero en catalogar como familia las uniones de hecho; asimismo, diversa legislación considera para realizar actos declarativos a las uniones de hecho y no solamente a las uniones matrimoniales. De este modo, las uniones de hecho, afirma Plácido, A. F. (2002) “...en un contexto social cambiante, se han abierto paso para quedarse y ser reconocidas...".

Las uniones de hecho a través de su incorporación en la Constitución de 1979 supuso un gran avance, más aún consagrar su regulación en el Código Civil de 1984, lo que fue seguido en este aspecto por la constitución de 1993. Sin embargo, poco se ha avanzado en el aspecto de su probanza, dejando a los concubinos, y a la parte más débil de los concubinos, la probanza de dicha situación; y es aquí precisamente que el Derecho ha sido indiferente, dejando a los integrantes de la relación con los medios establecidos en la legislación procesal civil la función de acreditar la relación convivencial. De allí que siendo un tipo de familia generalizado es menester regular su forma de probanza de mejor manera.

En nuestro país la informalidad es la normalidad, la excepción es que se cumplan las reglas. $Y$ en el ámbito de las relaciones de pareja y familiares la frecuencia con la que se presentan las uniones de hecho está dando como resultado que ésta se haya convertido en la regla y no como era antiguamente, la excepción. Esta realidad es producto de los cambios culturales, las ingentes migraciones ha convertido a nuestro país en un país de migrantes, personas que han dejado detrás sus pueblos con costumbres más conservadoras, más tradicionales $y$ se han adherido a los modelos urbanos que están en constante cambio, desde una adopción de modelos culturales de las zonas de origen de los migrantes y también del extranjero, influenciados básicamente por la televisión y los otros medios de comunicación.

Peralta, J. R. (2002), afirma que el matrimonio, considerado antiguamente la única forma de familia en la que se podía concebir a la familia ha pasado a ser solo una especie de ésta, pues las continuas modificaciones de los patrones culturales en nuestros países latinoamericanos, en el mundo en general, han dado como resultado que el concepto de familia se haya extendido a otros tipos ya no del tipo nuclear como se entendía desde la perspectiva eclesiástica sino que, se incorpore a este concepto tales como las familias ensambladas conformada solo por hermanos, tíos-sobrinos, abuelos nietos, madre-hijos, padre- hijos y una diversidad de formas que han sido reconocidos por la doctrina e inclusive por la legislación y hasta por el propio Tribunal Constitucional.

Nuestra legislación civil ampara las uniones de hecho y se ha encargado de establecer las pautas dividiendo a 
las uniones de hecho en las que cumplen con los requisitos legales para ser reconocidas judicialmente y las que no los cumplen tales requisitos.

La finalidad ha sido brindar protección jurídica a las uniones de hecho entre un varón y una mujer, sin impedimento matrimonial que potencialmente podrían convertirse en matrimonio.

Tenemos que el Código Civil de 1852 no reguló las uniones de hecho porque adoptó la doctrina del Código Canónico sobre el matrimonio, es decir proscribía las uniones de hecho por considerar que se vivía en pecado dado que no se consagraba el sacramento del matrimonio, obviamente esta concepción moralista fue adoptada por nuestra legislación civil y aun adoptándose decisiones judiciales en ese sentido hasta muy reciente.

Para el Código Civil de 1936 se consideraba a la unión de hecho como una sociedad de hecho en la que el hombre y la mujer conservan su independencia social y económica, no constituyendo una sociedad como el matrimonio en la que sí están vinculados en dichos aspectos. En materia constitucional, verificamos que la Constitución de 1979 en su artículo $9^{\circ}$ reconocía a la unión de hecho como aquélla "unión estable de un varón y una mujer, libres de impedimento matrimonial, que forman un hogar de hecho por el tiempo y en las condiciones que señala la ley, da lugar a una sociedad de bienes que se sujeta al régimen de la sociedad de gananciales en cuanto es aplicable. Similar fórmula fue repetida por la Constitución de 1993 en su artículo $5^{\circ}$ establece: "La unión estable de un varón y una mujer, libres de impedimento matrimonial, que forman un hogar de hecho, da lugar a una comunidad de bienes sujeta al régimen de la sociedad de gananciales en cuanto sea aplicable".

El Código Civil vigente, nacido al amparo de la Constitución de 1979 establece a través del artículo $326^{\circ}$ que "la unión de hecho voluntariamente realizada y mantenida por un varón y una mujer, para alcanzar finalidades $y$ cumplir deberes semejantes a los del matrimonio, origina una sociedad de bienes que se sujeta al régimen de la sociedad de gananciales, en cuanto le fuera aplicable, siempre que dicha unión haya durado por lo menos dos años continuos".

Asimismo la Ley $\mathrm{N}^{\circ}$ 26662, Ley de Competencia Notarial en Asuntos No Contenciosos, la misma que ha sido ampliada por la Ley $N^{\circ} 29560$ (16 de julio de 2010) autoriza a los Notarios a tramitar el reconocimiento de la unión de hecho contemplada en el artículo 326 del Código Civil. Así, se autoriza el reconocimiento notarial de aquellas uniones de hecho que hayan generado una comunidad de bienes, sujetas al régimen de sociedad de gananciales, en cuanto le sea aplicable, incorporando el título VIII sobre Declaración de Unión de Hecho a la referida Ley de Competencia Notarial en Asuntos No Contenciosos y se establece la inscripción de la declaración de la unión de hecho y su cese en el Registro Personal.

Por lo que se ha ampliado implícitamente el artículo 2030 del Código Civil, que regula los actos inscribibles en el Registro Personal, al 
permitir la incorporación a dicho Registro el reconocimiento notarial de las uniones de hecho y su cese $y$, por ende el acceso del reconocimiento judicial de las uniones de hecho y que inscritas las uniones de hecho también corresponde inscribir su cese en virtud a las causales reguladas en el artículo 326 del Código Civil (tercer párrafo) señala que la unión de hecho cesa por muerte, ausencia, mutuo acuerdo o decisión unilateral.

El Tribunal Constitucional en la sentencia del Expediente $\mathrm{N}^{\circ}$ 498-99AA/TC ha establecido que las uniones de hecho, a que se refiere el artículo 326 del Código Civil, le son aplicables el régimen de sociedad de gananciales, en cuanto sea pertinente, y, por tanto, con relación al caso (analizado) de transferencia de un inmueble por parte de uno de los convivientes estima que "...la disposición de los bienes que la conforman debe efectuarse de conformidad con lo estipulado en el primer párrafo del artículo $315^{\circ}$ del Código Civil, según el cual: "Para disponer de los bienes sociales o gravarlos, se requiere la intervención del marido y la mujer [..]. Dicho dispositivo debe ser interpretado de manera concordante con lo estipulado en el artículo $971^{\circ}$ del citado cuerpo normativo, cuyo texto establece que, existiendo copropiedad "las decisiones sobre el bien común se adoptarán: 1.Por unanimidad para disponer, gravar o arrendar el bien [...]..."

Si bien es cierto que la inscripción notarial y registral de las uniones de hecho son un avance en la dirección de crear una certidumbre, seguridad jurídica sobre este tipo de uniones, también es cierto que este trámite oneroso e igualmente dilatado como el matrimonio, genera en las personas que viven en una unión de hecho determinada desconfianza o desalientan su registro.

La carencia de este registro ha producido que aquellas parejas que tienen una relación concubinaria se vean desprotegidas en cuanto al aspecto patrimonial pues al constituir un patrimonio conjunto éste no necesariamente se refleja en comprobantes de pago por los bienes que ambos adquieren, pues siempre es registrado a nombre de uno de ellos y como no existe una fecha cierta de inicio de la relación concubinaria podría suceder eventualmente que alguno de ellos alegue sin justa razón que determinados bienes fueron adquiridos en fecha anterior al inicio de la relación concubinaria, de esta manera se deja desprotegido a uno de los concubinos, que generalmente es el que no tiene el manejo o administración de la economía del hogar y que por lo mismo es el principal adquirente de los bienes de la pareja y que finalmente figura en los comprobantes de pago.

Este problema de la falta de registro concubinario no sólo es un problema de tipo legal sino que además se convierte en uno de tipo moral, pues quienes se oponen al mismo sostienen que sería legalizar una irregular situación que es una situación de hecho para convertirla en una de derecho de forma definitiva, cuando la situación definitiva es el matrimonio.

Los Registros Jurídicos y el Índice Nacional del Registro Personal que forman parte del Sistema Nacional de los Registros Públicos, creemos que 
deberían incorporar la información que otra institución más cercana a la población como son las municipalidades, las que podrían estar brindando este registro. Municipalidades, como la de Chiclayo, Breña y el Callao a través de sus respectivas ordenanzas municipales han incorporado a su estructura orgánica los Registros de las Uniones de Hecho, pero sólo son una pequeña isla en este inmenso mar de la población peruana, dado que la municipalidad no creemos que se deba convertir en una promotora de las uniones de hecho sino de su registro como institución más cercana a la población y que se encuentra a través de las DEMUNAs y otras unidades orgánicas de la problemática de la población.

En el Perú se podría adoptar un modelo similar al derecho español (Lázaro, I. 1999), en cuanto a la inscripción de la unión de hecho en registros administrativos municipales, pues allá los ayuntamientos tienen esa facultad y no sólo registros de uniones de varones y mujeres sino hasta del mismo sexo, aunque nuestro país sólo deberían limitarse a las parejas heterosexuales. Como señalamos anteriormente de manera singular hasta el momento tres municipalidades han creado el registro de la unión de hecho y promoción de acceso al matrimonio, en el cual se pueden inscribir los convivientes.

\section{Materiales y métodos}

Para investigar los casos se ha empleado la técnica de la encuesta. $Y$ el instrumento para obtener los datos requeridos para llevar a cabo el desarrollo de la investigación fue obtenido a través de cuestionarios.

La muestra fue tomada durante el mes de febrero del 2013 en la ciudad de Tacna, desde del 02 al 26 de febrero.

A través de un cuestionario se obtuvo la opinión de abogados (litigantes, fiscales, jueces) que han tenido que ver con casos de unión de hecho para determinar los efectos de las normas de la unión de hecho. El tamaño de la muestra se determinó a través de la fórmula estadística y la selección de las unidades de análisis se realizó a discreción de la investigadora, dentro de estos profesionales del Distrito Judicial de Tacna. Esta muestra fue discrecional, totalizando 70 unidades de análisis. La información fue obtenida a través de las Encuesta realizadas a profesionales que han conocido, directa o indirectamente, casos de uniones de hecho, principalmente, abogados independientes.

Asimismo, para determinar los efectos de las normas de concubinato se obtuvo la opinión de personas que mantienen una relación convivencial. El tamaño de la muestra se determinó a discreción de la investigadora, siendo esta muestra determinada por la investigadora, totalizando las 80 unidades de análisis.

La investigación corresponde al tipo de investigación aplicada, debido a que tiene como propósito modificar parte del ordenamiento jurídico, por tanto la finalidad de la investigación es tratar de proporcionar elementos para modificar la realidad. El diseño de investigación, 
por su ubicación en el tiempo, es de tipo transversal.

Esta es una investigación socio jurídica, pues en su esencia está constituida por la interrelación que ocurre entre la realidad social donde se produce una realidad verificable estadísticamente, que es el incremento de uniones de hecho; y la realidad normativa que pretende regularla desde sus distintos ángulos, pues existe una problemática en la realidad que enfrentan las uniones de hecho en las zonas urbanas de Tacna y sus derechos normados en la Constitución Política del Perú, los que en su mayoría no se cumplen. Por lo tanto, esta problemática, que es una realidad empírico social, debe ser contrastada con el universo normativo, y entendida, para poder construir las fórmulas normativas resolutivas del problema.

\section{Resultados}

Para la determinación de la incidencia de las normas de inscripción registral de las uniones de hecho en la afectación de los derechos patrimoniales se ha procedido a realizar la recolección de los datos a nivel del Distrito Judicial de Tacna.

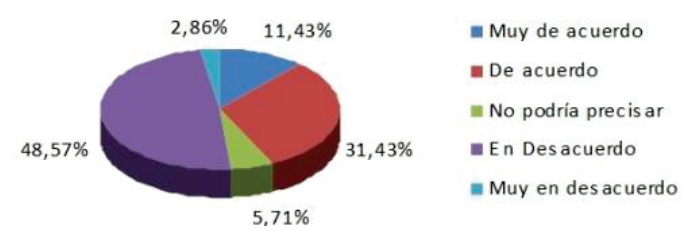

Figura 1.

Es adecuada la regulación de la norma (Código Civil) sobre el aspecto de la TUTELA de los derechos patrimoniales que se derivan de las uniones de hecho.
El $54.28 \%$ de los abogados señala estar en desacuerdo 48.57\%; y en Muy en Desacuerdo, el $2.86 \%$ respecto de la afirmación que sí es adecuada la regulación en nuestra legislación civil respecto de la protección de los derechos patrimoniales de los concubinos. Por lo que, en ese sentido, esta mayoría no cree que se encuentre adecuadamente regulada esta tutela o protección que deben tener las personas que mantienen una relación concubinaria (Figura 1).

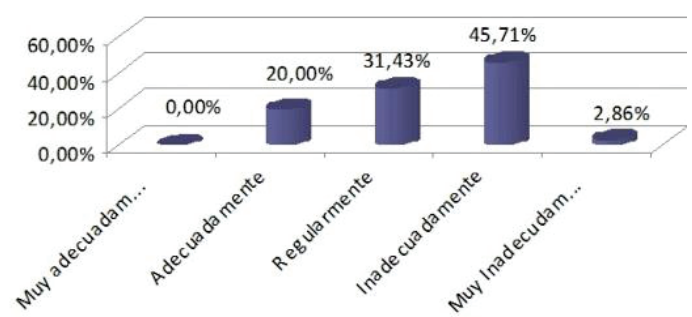

Figura 2.

Considera que la norma sobre inscripción en el registro de uniones de hecho en registros públicos cumple con su función de atraer a la mayoría de parejas en unión de hecho.

Solo el $20 \%$ de los abogados considera que las normas sobre Inscripción en el Registro de las Uniones de Hecho en Registros Públicos cumple con la función de atraer a la mayoría de parejas en unión de hecho; mientras que el $31.43 \%$ considera que lo hace regularmente; el $48.57 \%$ no lo considera adecuado; el $45.71 \%$ considera que lo hace inadecuadamente; y el $2.86 \%$ Muy inadecuadamente. Esto sería porque la imagen de Registros Públicos en nuestro país es de una institución que dificulta los trámites o que exige una serie de requisitos y que es un incentivo para la formalización (Figura 2). 


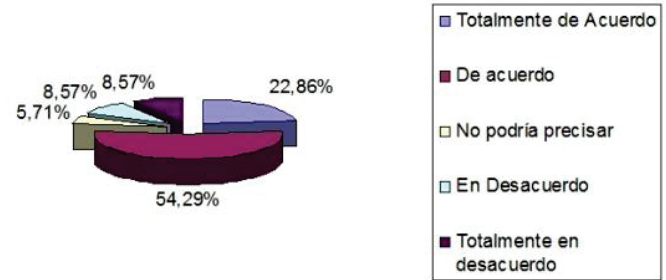

Figura 3.

Considera que la inscripción en el registro de las uniones de hecho debiera realizarse a través de municipios.

El $77.15 \%$ de los abogados encuestados considera que la inscripción en el Registro de las Uniones de Hecho debiera realizarse a través de los municipios. Ello se debería básicamente a la imagen que se tiene del Municipio, que es una institución cercana a la población y a la cual los ciudadanos recurren con mayor frecuencia que a los Registros Públicos, a los cuales han recurrido una vez en su vida, en muchos casos (Figura 3)
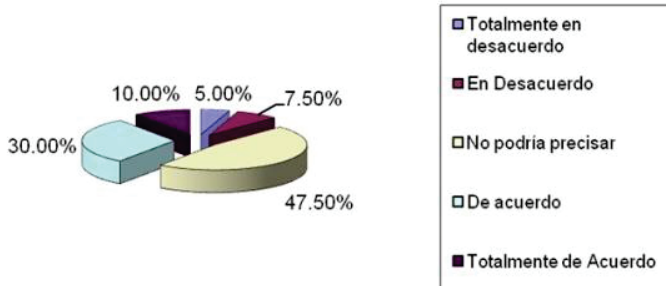

Figura 4.

Considera que el patrimonio de las parejas en uniones de hecho están plenamente protegidas en la forma que se encuentran regulado el Código Civil.

Frente a la pregunta de que si considera que el patrimonio de las parejas en uniones de hecho están plenamente protegidas en la forma que se encuentran regulado el Código Civil, el $47.50 \%$ de los ciudadanos señaló que no podría precisar. Esto supone un alto número de encuestados que no sabe realmente cómo se encuentra regulado el concubinato en nuestra legislación civil. Solo el $12.50 \%$ está en desacuerdo y muy en desacuerdo con la forma en que se encuentra legislado. Un llamativo $40 \%$ sí mostró estar de acuerdo y muy de acuerdo en la creencia que la ley si protegería el patrimonio de los integrantes de la relación convivencial (Figura 4).

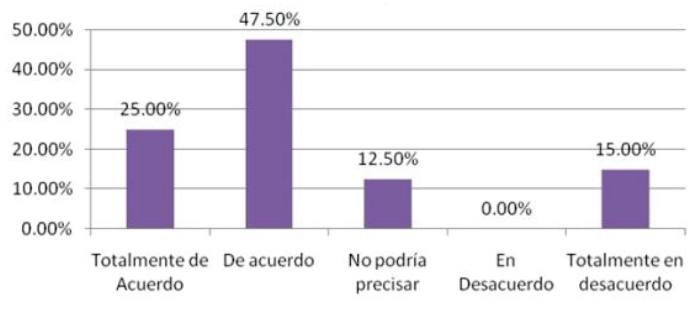

Figura 5

Considera que la inscripción en el registro de las uniones de hecho debiera realizarse a través de los municipios.

El $72.50 \%$ de los ciudadanos y ciudadanas encuestadas considera que la inscripción en el Registro de las Uniones de Hecho debiera realizarse a través de los municipios. Ello se debería básicamente a la imagen que se tiene del Municipio, que es una institución cercana a la población. Mientras que el $15 \%$ se mostró en total desacuerdo. Si vemos más arriba las respuestas de los profesionales, más del $77 \%$ está de acuerdo con que este tipo de inscripciones las hagan los municipios, lo que supone una confianza aún mayor de los profesionales que de los ciudadanos en los municipios. Dado el nivel de información que ellos manejan en relación con la que manejan los ciudadanos o de la percepción que tienen los ciudadanos respecto de su ente local (Figura 5).

\section{Discusión}

Debe apreciarse que la unión de hecho siempre tiene un margen de inseguridad que es lo que se ve reflejado en las respuestas dadas por 
los encuestados, puesto que en ese sentido no se va a equiparar a la seguridad patrimonial en un régimen de gananciales que puede otorgar un matrimonio, lo que se ve reflejado en las respuestas de los hombres y mujeres de Derecho al responder esta pregunta.

En la indagación realizada en las notarías de Tacna se encuentra que no existen trámites de registro de uniones de hecho de acuerdo de la norma habilitante para ello, por tratarse de una norma no tan necesaria. Si es que se la examina desde la perspectiva del costo beneficio, las parejas prefieren permanecer en estado de unión de hecho que acudir a una notaría y luego a registros públicos para inscribir su unión de hecho; los costos de por sí ya son desalentadores y los trámites lo son aún más. De esta forma se verifica la ineficacia de estas normas pues no ha solucionado los problemas que pretendía resolver.

Las Municipalidades son entidades cercanas al ciudadano. No es difícil ver que municipalidades existen en todos los rincones de nuestro país, mientras que los registros públicos y notarías únicamente existen en las principales ciudades, por lo que existe la posibilidad de que los municipios pudieran realizar este trámite de uno de sus vecinos, que es así como se trata al administrado en las municipalidades; mientras que para Registros Públicos son denominados usuarios (o consumidores de servicios). Desde allí la diferencia entre que la población se sienta más cercana a su municipalidad que a la Oficina de Registros Públicos.
En cuanto a la percepción o conocimiento de sobre si las normas del Código Civil protegen el patrimonio de los concubinos, los profesionales consideran con más conocimiento.

Se puede verificar que la población encuestada considera que las municipalidades se han convertido en instituciones cercanas al vecino y por tanto son entes idóneos para llevar a cabo los registros de las uniones de hecho, más aún cuando existe una oficina de registro civil en ellas.

\section{Conclusiones}

- La regulación del régimen patrimonial de la unión de hecho en la legislación nacional carece de integralidad y precisión, puesto que los operadores del Derecho mayoritariamente creen que no existe correspondencia de las normas de nuestra legislación sustantiva civil con la realidad social. Así como, otro porcentaje importante cree que la norma no es precisa, pues contendría ambigüedades o imprecisiones que afectan los derechos patrimoniales.

- El bajo nivel de conocimiento de las parejas de concubinos de la posibilidad de inscripción de su relación en los Registros Públicos y lo costoso y dificultoso del procedimiento propicia su no registro; puesto que un importante segmento no considera adecuadas las normas sobre Inscripción en el Registro de las Uniones de Hecho en Registros Públicos ni que estas cumplan con su función de atraer a la mayoría de parejas en unión de hecho. Asimismo, una mayoría 
considera que uno de los factores para que las uniones de hecho no se registren es porque esos trámites son costosos y dificultosos.

- Se afectan los derechos patrimoniales y su seguridad económica de los concubinos y sus familias al no estar incorporados en un registro municipal de uniones de hecho, pues se ha demostrado que los operadores del Derecho mayoritariamente señalan que las normas sobre protección de los aspectos patrimoniales en las uniones de hecho no son integrales y además las normas no son precisas.

- Existe una relación significativa entre la falta de un registro de las parejas que mantienen una unión de hecho y la vulneración de los derechos fundamentales patrimoniales de los concubinos. Por cuanto, como se verifica la falta de precisión, de integralidad, de precisión de límites en la determinación del inicio del tiempo para el reconocimiento de la relación de unión de hecho, conspira contra los derechos patrimoniales.

- Se debe promulgar la norma que permita la creación del Registro de inscripción de las parejas que mantienen una relación convivencial e inscribir dicha relación en las Oficinas de los Registros Civiles de las Municipalidades.

\section{Referencias Bibliográficas}

1. Cornejo, M. T. (2000). Matrimonio y Familia: Su tratamiento en el derecho. Lima: Ediciones Tercer Milenio S.A..

2. Plácido, A. F. (2002). Regímenes patrimoniales del matrimonio y las uniones de hecho en la doctrina y en la jurisprudencia (1a ed.). Lima: Ediciones Gaceta Jurídica.

3. Peralta, J. R. (2002). Derecho de Familia en el Código Civil. Capítulo V. (3a ed.). Perú: Ediciones Moreno S.A.

4. Lázaro, I. (1999). Las Uniones de hecho en el Derecho Internacional Privado Español. Madrid: Ediciones Tecnos.

E-mail: smirandaperez@hotmail.com

Recibido: 02/04/14

Aceptado para publicación: 27/05/2014 